\title{
Data Fusion of Power and Time Measurements for Mobile Terminal Location
}

\author{
Michael McGuire, Member, IEEE, Konstantinos N. Plataniotis, Senior Member, IEEE, and \\ Anastasios N. Venetsanopoulos, Fellow, IEEE
}

\begin{abstract}
The location of mobile terminals in cellular networks is an important problem with applications in resource allocation, location sensitive browsing, and emergency communications. Finding cost effective location estimation techniques that are robust to Non-Line of Sight (NLOS) propagation, quantization, and measurement noise is a key problem in this area. Quantized time difference of arrival (TDoA) and received signal strength (RSS) measurements can be made simultaneously by CDMA cellular networks at low cost. The different sources of errors for each measurement type cause RSS and TDoA measurements to contain independent information about mobile terminal location. This paper applies data fusion to combine the information of RSS and TDoA measurements to calculate a superior location estimate. Nonparametric estimation methods, that are robust to variations of measurement noise and quantization, are employed to calculate the location estimates. It is shown how the data fusion location estimators are robust, provide lower error than the estimators based on the individual measurements, and have low implementation cost.
\end{abstract}

Index Terms-Nonparametric statistics, wireless sensor networks, location-dependent and sensitive mobile applications, location estimation.

\section{INTRODUCTION}

$\mathrm{M}$ OBILE terminal location is an important problem for modern cellular networks. The knowledge of mobile terminal location can be used to enhance resource allocation in the cellular network, enable location sensitive information browsing, as well as helping users making emergency communications [1], [2].

The characteristics of the radio channel between the mobile terminal and fixed location base stations are dependent on the location of the mobile terminal. This allows for the location of the mobile terminal to be estimated from measurements made of the radio propagation channels. The most popular proposed measurements are Received Signal Strength (RSS), Angle of Arrival (AoA), Time of Arrival (ToA), and Time Difference of Arrival (TDoA)[3].

AoA measurements with the desired accuracy require special antennae, which are not presently used in any currently deployed cellular network standard. To obtain ToA measurements, the receivers must have knowledge of the transmission times of the radio signals. To give them this knowledge with the accuracy required is very expensive [3]. RSS and TDoA measurements, however, can be implemented in third generation cellular networks at low cost. Location of mobile terminals using only RSS measurements or only TDoA measurements has been discussed in the radiolocation

- M. McGuire is with the Department of Electrical and Computer Engineering, University of Victoria, PO Box 3055 STN CSC, Victoria, BC, V8W 3P6, Canada. E-mail: mmcguire@ece.uvic.ca.

- K.N. Plataniotis and A.N. Venetsanopoulos are with The Edward S. Rogers Sr. Department of Electrical and Computer Engineering, University of Toronto, 10 King's College Road, Toronto, Ontario M5S $3 G 4$.

E-mail: \{kostas,anv\}@dsp.toronto.edu.

Manuscript received 2 May 2003; revised 27 Nov. 2003; accepted 4 Jan. 2004; published online 27 Jan. 2005.

For information on obtaining reprints of this article, please send e-mail to: tmc@computer.org, and reference IEEECS Log Number TMC-0059-0503. literature [4], [5], [6]. The use of the GPS navigation system has also been proposed for location of mobile terminals [7]. However, the GPS system was not designed for operation in the urban environments of greatest interest to cellular network providers and its performance can be degraded in these cases [8].

It is not certain if the use of TDoA, RSS, or GPS measurements alone can achieve the desired location accuracies. This paper discusses methods to use RSS and TDoA measurements simultaneously to create location estimates with lower location errors than the estimators using either measurement type alone. The data fusion methods introduced in this paper increase the accuracy of mobile terminal location without expensive system modifications such as requiring more base station measurements or increasing the measurements' resolutions. GPS, which requires the addition of a GPS receiver in each mobile terminal, is not considered explicitly in this paper. However, GPS systems are based on ToA and TDoA measurements so the results contained within this paper for TDoA measurements give information relevant for a location system that combines GPS and RSS measurements together to increase location accuracy in urban streets or other areas where GPS alone might not be sufficiently accurate.

These new estimators are designed to be robust to difficult radio propagation conditions. Accurate mobile terminal location estimation is trivial for Line of Sight (LOS) radio propagation (defined by the shortest distance straight line paths between the mobile terminal and base stations being unobstructed) when the measurement noise is negligible and the measurements have high resolution [3]. In actual location estimation applications, Non-Line of Sight (NLOS) propagation, when obstructions block the shortest distance propagation paths from mobile terminal to the base stations, is common, particularly in urban areas. As well, RSS and TDoA measurements made in 
cellular networks contain additive noise created by radio interference and receiver noise as well as nonlinear noise created by multipath propagation. High resolution of path loss measurements can be made with expensive hardware in the receivers and high resolution time delay measurement are possible with computationally expensive superresolution signal processing algorithms [9], [10], [11]. The resolution of measurements in actual cellular networks is determined by the resources that the system designers are willing to assign to the location system. The robustness of a location algorithm with respect to low resolution measurements is a key factor to its ultimate success in real networks.

The authors have previously demonstrated location estimation algorithms using RSS, ToA, or TDoA measurements that are robust to additive measurement noise and NLOS propagation [12], [13]. These algorithms are based on the use of nonparametric estimation techniques which use survey data taken from the propagation environment to construct approximate joint probability density functions (pdfs) of the measurements and mobile terminal location. With these approximate pdfs, Bayesian estimation is used to calculate mobile terminal location estimates. The application of similar nonparametric estimation techniques has been shown to provide excellent results during field tests in complex indoor environments [14], [15]. Accurate nonparametric location estimation based on high-resolution measurements of the multipath delay profiles has also been demonstrated for a simulated complex outdoor urban environment [16].

The measurement noise for TDoA and RSS measurements come from fundamentally different sources which suggest that the errors in the mobile terminal location estimates for each measurement type are at least partially independent. Data fusion techniques exploit the independence between different measurements to create estimators that have higher accuracy than the estimators based on single measurement types [17].

Previous work has explored the data fusion of ToA/ AoA, TDoA/AoA, or ToA/TDoA/AoA measurements [18], [19], [20], [21], [22]. The disadvantage of these methods is that AoA measurements require specialized antennae which makes these methods inapplicable to currently deployed cellular networks. This paper explores the data fusion of RSS and TDoA measurements, measurement that can be made simultaneously without requiring additional hardware in the existing digital CDMA terminals. Data fusion at both the estimate and measurement level are compared to find the optimal fusion technique in terms of computational cost and estimator accuracy. For data fusion at the estimate level, location estimates are calculated for RSS and TDoA measurements individually. Weights are calculated for the estimate of each measurement type based on the estimate's error covariance. These weights are used to linearly combine the individual estimates together into an estimate with superior accuracy. Data fusion at the measurement level uses a single measurement vector that has both the RSS and TDoA measurements. A single estimation calculation using the combined measurement vector computes the estimated location. The advantages and disadvantages of each of these data fusion techniques are listed.
TABLE 1

Measurement Equations

\begin{tabular}{ccc}
\hline & Path Loss Measurement & TDoA Measurement \\
\hline Raw Measurement & $\tilde{\boldsymbol{p}}=\mathrm{p}(\boldsymbol{\theta}, \boldsymbol{b})+\boldsymbol{\varepsilon}_{p}$ & $\tilde{\boldsymbol{\tau}}=\mathrm{d}(\boldsymbol{\theta}, \boldsymbol{b})+\boldsymbol{\varepsilon}_{\tau}$ \\
True Measurement & $\boldsymbol{p}=\operatorname{rnd}\left(\frac{\tilde{\boldsymbol{p}}}{q_{p}}\right) \cdot q_{p}$ & $\boldsymbol{t}=\operatorname{rnd}\left(\frac{G \tilde{\tau}}{q_{t}}\right) \cdot q_{t}$ \\
\hline
\end{tabular}

The location algorithms are evaluated using a simulated urban microcell radio propagation environment. The regions of greatest interest to cellular network providers are urban regions. These regions generate the most revenue and also have complicated radio propagation environments with high probabilities of NLOS radio propagation.

Section 2 describes the signal model assumed by the estimation procedure. The simulated signal model used to evaluate the proposed location methods is also fully described. Section 3 describes the proposed estimation procedures. Section 4 describes the results of simulations used to evaluate the location procedures. The robustness of the new location methods to changes in the location environment and measurement noise are demonstrated. Section 5 gives the conclusions of the paper and lists some possible avenues for future research in this area.

\section{Signal Model}

The true location of the mobile terminal is denoted $\boldsymbol{\theta}=(x, y)$, where $(x, y)$ is the location of the mobile terminal in two-dimensional space. This paper will only consider a two-dimensional location, but the methods are easily extended to three dimensions. An index vector $b$ contains the indices of the base stations making measurements to locate the mobile terminal. Two types of measurements are used in this paper: RSS path loss measurements, denoted $p$ and TDoA measurements, denoted $t$.

If there is sufficient diversity in the bearing directions to the measuring base stations from the mobile terminal, the measurement noises for each base station are independent. Without this diversity, there will be some dependence in the measurement noise of different base stations. However, It has been shown that, without this bearing angle diversity, there is a degradation in location accuracy, named in the literature as Geometric Dilution of Precision (GDOP), even if the measurement noises for each base station are independent in this case [23]. This can be avoided with a proper measuring base station selection algorithm. The hand off algorithm, which determines which base station is communicating with the mobile terminal, provides the network with probabilistic knowledge of the area in which the mobile terminal resides. Using this knowledge, the base station selection algorithm ensures that the measuring base stations are likely to have good bearing angle diversity with respect to the mobile terminal [12].

The RSS path loss measurements are given in decibels. Time measurements are converted to distance measurement by multiplication by the speed of light. For a given selection of measuring base stations $b$, the general form of the measurement equations is given in Table 1 . The raw measurement equation is the preliminary form of path loss or time measurements that would be available if the 
measurement device had infinite precision and had knowledge of the exact transmission times. For the path loss measurements, $\mathrm{p}(\boldsymbol{\theta}, \boldsymbol{b})$ is a vector function which gives the deterministic path loss values from the base stations indicated in $b$ to the mobile terminal location $\theta$, and $\varepsilon_{p}$ is a random vector containing the random portion of the path loss and RSS measurement noise. The form of the $\mathrm{p}(\cdot)$ function is highly dependent on the propagation environment. The vector function $d(\theta, b)$ returns the lengths of the shortest unobstructed propagation paths from the mobile terminal location $\theta$ to the measuring base stations, $b . \varepsilon_{\tau}$ is the time measurement error vector which includes the effects of synchronization error in the receivers, scatterers, and multipath propagation. The effects of NLOS propagation are split into two categories. The effects of NLOS propagation caused by large nonmoving objects such as buildings or geographic features blocking propagation paths will be included in $\mathrm{d}(\boldsymbol{\theta}, \boldsymbol{b})$ as they are deterministic given mobile terminal location. The effects of NLOS propagation caused by small moving obstacles such as vehicles and pedestrians are included in $\varepsilon_{\tau}$ since they are nondeterministic given mobile terminal location.

The true measurement equations in Table 1 reflect the RSS path loss and TDoA measurements that are available to a real-world measurement device without knowledge of transmission times and only finite precision. Quantization is modeled using the vector function defined as $y=\operatorname{rnd}(x)$, where the $k$ th entry of $y$ is the closest integer value to the $k$ th entry of $x$. The constants $q_{p}$ and $q_{t}$ specify the quantization levels. A single TDoA measurement for two base stations consists of the difference of the time measurements for the base stations. The difference matrix $G$ is used to denote the difference used to calculate each entry of the TDoA measurement vector $t$. Each row of $G$ contains a single one and a single minus one with all other entries being zero. The measurement equations for TDoA from Table 1 can also be use to specify a hybrid ToA/TDoA measurement scheme. If $G$ is full rank or, equivalently, all ToA measurements are used to create the maximum number of linearly independent TDoA measurements and the number of measuring base stations is $m$, then $t$ is of length $m-1$.

Of primary consideration for the application of data fusion of RSS path loss and TDoA measurements is the relationship between and $\varepsilon_{p}$ and $\varepsilon_{\tau}$. If these vectors were perfectly dependent, i.e., knowledge of $\varepsilon_{p}$ gives complete knowledge of $\varepsilon_{\tau}$, then data fusion of RSS and TDoA would result in no improvement in estimator accuracy as, ignoring quantization effects, both measurement types give identical information about the mobile terminal location. The radio propagation literature suggests that there is a relationship between the measurement noise of the two measurement types but this relationship has not been fully characterized [24]. Propagation surveys indicate that there is a partial correlation between path loss measurement noise variance and the median value of excess propagation delay in urban environments [25]. Radio propagation path loss is determined by the sum of the received signal power over all propagation paths between the base station and the mobile terminal. A TDoA measurement is determined only by the propagation delays of the shortest propagation paths from

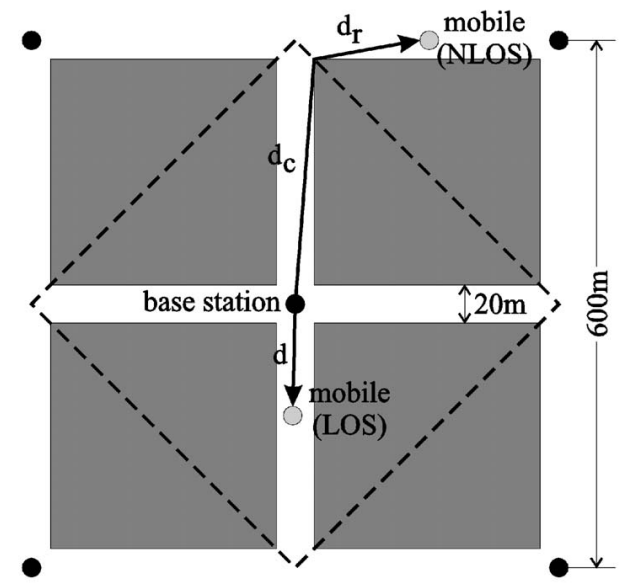

Fig. 1. Manhattan propagation model (single cell).

the involved base stations to the mobile terminal. The discrepancy between the aspects of propagation engaged in each measurement type ensures that there is some independence between the measurement noise for each measurement type.

The new location method is evaluated using a simulated radio propagation environment. An urban environment is simulated since these are the regions of greatest interest to cellular network designers. The simulated radio propagation model is described in the next section.

\subsection{Simulation Signal Model}

For a given mobile terminal location $\theta$, the generated signal vectors are $(p, t, b)$, where $p$ is the vector of path loss measurements, $t$ is the vector of TDoA measurements, and $b$ is a vector of the indices of the base stations that are the source of measurements.

The radio signal model used to evaluate the estimation algorithm is based on an urban radio propagation model used to evaluate other radio location methods [3]. The simulation model includes a model for simulated radio propagation into buildings [26]. The layout of the cell for a single base station's cell in this urban environment is shown in Fig. 1, where the shaded areas represent buildings.

From Table 1, it can be seen that to simulate path loss and TDoA measurements the generation methods for the deterministic portions of the propagation models, $p(\boldsymbol{\theta}, \boldsymbol{b})$ and $\mathrm{d}(\boldsymbol{\theta}, \boldsymbol{b})$, and random portions of the propagation models, $\varepsilon_{p}$ and $\varepsilon_{\tau}$, need to be specified. The propagation values for each measuring base station must be calculated separately. We will denote as $[x]_{k}$ the $k$ th entry of the vector $x$. The equations for the deterministic propagation functions used in the simulations are provided in Table 2 . We specify the distance from the mobile terminal to the base station of interest as $d$. For NLOS propagation to mobile locations on the street, $d_{c}$ is the distance from the base station to the corner, and $d_{r}$ is the distance from the corner to the mobile terminal as shown in Fig. 1. In the case of NLOS propagation into a building, we assume that radio propagation inside a building for a radio signal originating outside of the building is mostly perpendicular to the external walls of the building [26]. The distance $S$ is the distance of radio propagation outside of the building. The distance $d_{\perp}$ is the perpendicular distance from the building 
TABLE 2

Propagation Models for Simulations

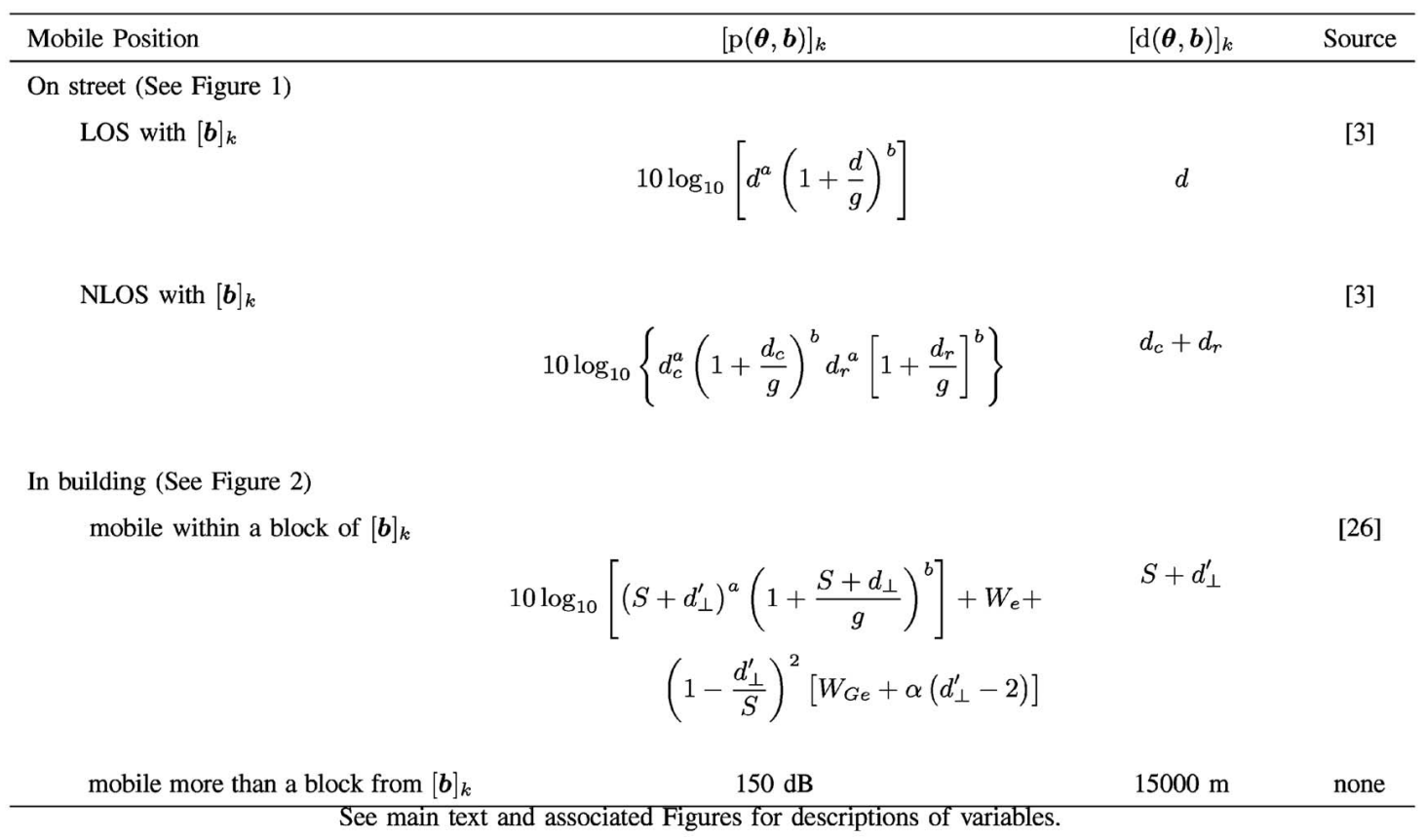

external wall the base station. The distance $d_{\perp}^{\prime}$ is the perpendicular distance from the building external wall to the mobile terminal. The distances $S, d_{\perp}$, and $d_{\perp}^{\prime}$ are illustrated in Fig. 2. $W_{e}$ is the attenuation factor of the exterior wall of the building when the signal path is normal to the wall's surface. $W_{G e}$ is an attenuation factor associated when the radio signal penetrating the external walls at nonperpendicular angles. $\alpha$ is the distance attenuation factor for radio signals traveling through a building. The values used for these constants in the simulations described in this paper are given in Table 3. It is assumed that a base station cannot provide a high quality radio signal to mobile terminals located in buildings more than a block away. The propagation model in Table 2 for this condition return extremely poor values for path loss and propagation distance to reflect this.

It has been shown that the random portion of the path loss for urban radio propagation, $\varepsilon_{p}$, can be well modeled as a Gaussian random variable assuming the mobile is moving and the effects of multipath fading on the RSS is removed by averaging over several seconds[27], [28]. This assumption is made in the simulations, with path loss error simulated as a Gaussian random variable with mean zero

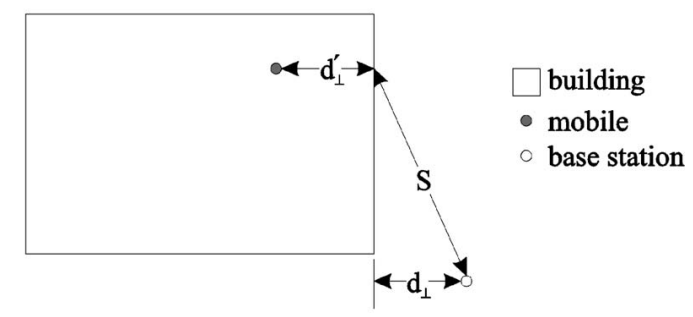

Fig. 2. Building propagation model. and standard deviation $\sigma_{p}$ varying from 2 to $8 \mathrm{~dB}$, which are typical values for urban propagation. The simulated path loss measurement noise for each base station is independent of the path loss measurement noise for other base stations. To simulate completely stationary users, the measurement noise would also have to include a random variable modeling the effects of multipath propagation on the received power.

The random portion of the propagation time measurements, $\varepsilon_{\tau}$, is the sum of two random processes: synchronization error, and multipath propagation from mobile scatterers. Synchronization error for CDMA receivers, which are likely to be used in next generation cellular

TABLE 3

Propagation Constants

\begin{tabular}{lc}
\hline Constant & Value \\
\hline$a$ & 2.0 \\
$b$ & 2.0 \\
$g$ & $150 \mathrm{~m}$ \\
$W_{e}$ & $10 \mathrm{~dB}$ \\
$W_{G e}$ & $20 \mathrm{~dB}$ \\
$\alpha$ & $0.6 \mathrm{~dB} / \mathrm{m}$ \\
$\sigma_{p}$ & $2.0-8.0 \mathrm{~dB}$ \\
$\sigma_{\tau}$ & $10.0-20.0 \mathrm{~m}$ \\
$q_{p}$ & $1.0 \mathrm{~dB}$ \\
$q_{t}$ & $60.0 \mathrm{~m}$ \\
$\lambda$ & $35.0 \mathrm{~m}$ \\
\hline
\end{tabular}


systems, has been shown to be well modeled as a Gaussian random variable [29]. Synchronization error in our simulations is a Gaussian random variable with zero mean and a standard deviation, $\sigma_{\tau}$, varying from 10 to $20 \mathrm{~m}$. We simulate the effects of scatters on the time measurements with an approximation to the "Urban" propagation model from [24], where the propagation delay for the earliest arriving multipath at a receiver from a transmitter is the smallest of 20 independent draws of a uniform random variable varying from 0 to $720 \mathrm{~m}$ and this random variable is independent of path loss measurement noise. To simplify simulation, this process is approximated with an exponential random variable of mean $\lambda=35.0 \mathrm{~m}$.

The time measurement error value is modeled as being independent between different base stations. The simulated synchronization noise variance is the same for all base stations. This is justified by simulations studies that show the synchronization error variance is nearly constant for a large range of receiver signal to noise ratios in urban microcell propagation [3].

The received signal power measurements are rounded to the nearest decibel, $q_{p}=1.0 \mathrm{~dB}$, to simulate the resolution that would be seen in true field implementations. The TDoA time measurements are quantized to the nearest $q_{t}=60 \mathrm{~m}$ interval to reflect quantization effects that would be seen in actual field implementations. The $60 \mathrm{~m}$ quantization interval was selected to approximately match the one quarter chip interval period in the IS-95 radio interface standard, which is a rough approximation of the time measurement quantization of radio receivers using this interface without using superresolution algorithms. All constants for radio propagation are given in Table 3.

The measurement vectors are truncated so that only those measurements from the three base stations with the lowest path loss measurements of the set of nine closest base stations are used to locate the mobile terminal. Since mobile terminals in actual cellular networks cannot make measurements for all the base stations in the network and use power measurements to decide which base stations to be associated with for their hand off algorithms, this truncation is a good approximation to actual field measurement practice. All TDoA measurements are calculated with respect to the time measurement for the base station with the lowest path loss measurement. The difference matrix $G$ is

$$
G=\left[\begin{array}{ccc}
1 & -1 & 0 \\
1 & 0 & -1
\end{array}\right],
$$

where the first entry of $b$ is the base station with the lowest path loss measurement.

\section{Estimation Methods}

The main focus of this paper is on data fusion for mobile terminal location estimation, the use of more than one measurement type to calculate the location of the mobile terminal in a cellular network. Specifically, we concentrate on the use of path loss and TDoA measurements.

The goal of this paper is to calculate the estimate of mobile terminal location, denoted $\hat{\boldsymbol{\theta}}$ with the minimum Mean Square Error (MSE) for the measurement vector $z$, i.e., the sum of the diagonal elements of $\mathrm{E}\left[(\hat{\boldsymbol{\theta}}-\boldsymbol{\theta})(\hat{\boldsymbol{\theta}}-\boldsymbol{\theta})^{T}\right]$ has the minimum value for all valid estimators of $\theta$ using $z$. It is well-known that the estimator that has these properties is the Minimum Mean Square Error (MMSE) estimator which is given as $\hat{\boldsymbol{\theta}}_{z}=\mathrm{E}[\boldsymbol{\theta} \mid z]$ [30].

Data fusion of the RSS and TDoA measurements at the estimation level is described in Section 3.1. Methods for estimating $\theta$ using either path loss or TDoA measurements are described along with methods of fusing the estimates together. Both parametric and nonparametric estimation methods are described with their respective advantages and disadvantages listed. Data fusion at the measurement level, estimation using a measurement vector consisting of both $p$ and $t$ in a single calculation, is presented in Section 3.2.

\subsection{Separate Estimation of $\theta$ for Each Measurement Type}

Estimation methods for calculating estimates of mobile terminal location using either path loss measurements or TDoA measurements is a fairly mature field. We consider MMSE methods that use prior knowledge of mobile terminal location that is available from the hand off algorithm or measuring base station selection data. Other techniques that do not use this data exist but do not provide comparable performance [12], [13].

If the MMSE estimation expectation of $\theta$ given measurement $z$ is expanded, the equivalent integral estimation equation is

$$
\hat{\boldsymbol{\theta}}_{z}=\int \boldsymbol{\theta} \mathrm{f}(\boldsymbol{\theta} \mid z) d \boldsymbol{\theta},
$$

where $\mathrm{f}(\boldsymbol{\theta} \mid z)$ is the conditional pdf of $\theta$ given $z$ and the integration is performed over the domain of $\boldsymbol{\theta}$. Using Bayes' rule and rearranging terms, we obtain:

$$
\hat{\boldsymbol{\theta}}_{z}=\frac{\int \boldsymbol{\theta} \mathrm{f}(z \mid \boldsymbol{\theta}) \mathrm{f}(\boldsymbol{\theta}) d \boldsymbol{\theta}}{\int \mathrm{f}(z \mid \boldsymbol{\theta}) \mathrm{f}(\boldsymbol{\theta}) d \boldsymbol{\theta}},
$$

where $\mathrm{f}(z \mid \boldsymbol{\theta})$ is the conditional pdf of $z$ given $\boldsymbol{\theta}$ and $\mathrm{f}(\boldsymbol{\theta})$ is the pdf of $\theta$. If the radio propagation environment is known, then $\mathrm{f}(z \mid \theta)$ is characterized by the pdf of the measurement noise for measurement $z$ and the model for the deterministic portion of $z$ given by the radio propagation model. Equation (3) shows that the MMSE estimator requires knowledge of the conditional density of the measurements given the location value $\theta$ and the prior pdf of $\theta$.

If we use the measurement protocol defined in Section 2.1, then the measurement $z$ is either $p$ or $t$ and the prior $\operatorname{pdf} f(\theta)$ is the conditional pdf of $\theta$ given the measuring base station selection index vector $\boldsymbol{b}, \mathrm{f}(\boldsymbol{\theta} \mid \boldsymbol{b})$.

Simple closed forms for $\mathrm{f}(z \mid \boldsymbol{\theta})$ and $\mathrm{f}(\boldsymbol{\theta} \mid \boldsymbol{b})$ for $z=\boldsymbol{p}$ or $\boldsymbol{t}$ only exist for simple propagation cases such as when all radio propagation is LOS [28]. Previous work has been performed on MMSE estimation using parametric estimation for path loss measurement vectors [31]. A parametric equation for the conditional pdf of $p$ given $\theta$ is used based on a LOS radio propagation model. The radio propagation parameters are learned from survey data taken from the propagation environment using the EM algorithm to handle data truncation from base station selection and quantization of the measurements. The advantage of the parametric method is that, if the parametric model closely matches the 
true environment, the MMSE equation in (3) can then be directly integrated to obtain high quality location estimates. The disadvantage is that if the propagation environment does not closely match the parametric model, the calculated estimates will have high errors. The regions of greatest interest to cellular network providers are urban regions which have plentiful NLOS propagation and do not conform to simple parametric models.

This paper proposes location based on nonparametric MMSE estimation. The nonparametric MMSE location methods are based upon the existence of survey data. The survey data consists of a set of locations with three measurement vectors for each location. The location of the $j$ th survey point is denoted $\boldsymbol{\theta}_{j}$. The measurement vectors of survey point $j$ are $\left(\boldsymbol{b}_{j}, \boldsymbol{p}_{j}, \boldsymbol{t}_{j}\right)$. $\boldsymbol{b}_{j}$ is a vector of base station indices which indicates which base stations made the measurements at the $j$ th survey point. $p_{j}$ is the vector of path loss measurements for the $j$ th survey point. $t_{j}$ is the TDoA measurement vector for the $j$ th survey point. The measurement process for the survey points are identical to measurement process used for the location measurement $(b, p, t)$ described in Section 2.1. In the cell for each base station are located $N=500$ survey points. The locations of these survey points are uniformly distributed over the cell area. Techniques on methods for bounding the number of required survey points is presented in [12]. Data surveys of path loss measurements are already performed in cellular networks for cell coverage confirmation [25], [32]. TDoA measurements of the resolution specified in this paper are not expensive to obtain, so the extra cost of collecting TDoA measurements during the measurement surveys would not be large.

The first step of nonparametric location estimation algorithms is to use the base station index vector, $b$, to identify the general area in which the mobile terminal is likely to be located. Using the measurement protocol defined in Section 2.1, $[\boldsymbol{b}]_{1}$ identifies the base station with the lowest path loss measurement to the mobile terminal. We assume that the mobile terminal is located either in the cell associated with base station $[\boldsymbol{b}]_{1}$ or in one of the cells adjacent to it. Using this prior location assumption, we collect the survey data for all survey points located in the cell for base station $[\boldsymbol{b}]_{1}$ and the immediately adjacent cells.

The survey set is then reduced to only the survey points relevant for location. Only survey points where $b_{j}$ matches $b$ are kept for the location algorithm. Matching is taken as meaning that the entries of $\boldsymbol{b}_{j}$ can be reordered to create a vector equal to $\boldsymbol{b}$. For example, if $\boldsymbol{b}=\left[\begin{array}{lll}1 & 2 & 3\end{array}\right]^{T}$, indicating that base stations 1,2 , and 3 are associated with the measurements in $\boldsymbol{p}$ and $\boldsymbol{t}$, then $\boldsymbol{b}_{j}=\left[\begin{array}{lll}3 & 1 & 2\end{array}\right]^{T}$ would match $\boldsymbol{b}$, while $\boldsymbol{b}_{k}=\left[\begin{array}{ll}3 & 4\end{array}\right]^{T}$ would not since $\boldsymbol{b}_{k}$ contains the entry 4 which is not in $b$. (The superscript $T$ denotes matrix or vector transpose.)

To properly use the survey points, for all valid $j$ and $k$, $\left[\boldsymbol{p}_{j}\right]_{k}$ and $[\boldsymbol{p}]_{k}$ must have measurements made by the same base stations. The same property is required for $\left[\boldsymbol{t}_{j}\right]_{k}$ and $[\boldsymbol{t}]_{k}$. The measurement vectors for survey points in which $\boldsymbol{b}_{j}$ matches $b$ but $b_{j} \neq b$ do not have this property and must be transformed as described in Appendix B, before they are used in the estimation process.
After the above survey set reduction to obtain valid survey points, a nonparametric MMSE estimation procedure is applied to calculate a mobile terminal location estimate. Full derivation and justification of the nonparametric estimation equations for use of the path loss and TDoA measurement vectors individually have been previously presented by the authors [12], [13]. The theory of nonparametric MMSE estimators is reviewed in Appendix A.

The location estimate based on path loss measurements is calculated as

$$
\hat{\boldsymbol{\theta}}_{p}=\frac{\sum_{j=1}^{n} \boldsymbol{\theta}_{j} \mathrm{~K}_{p}\left(\boldsymbol{p}-\boldsymbol{p}_{j}\right)}{\sum_{j=1}^{n} \mathrm{~K}_{p}\left(\boldsymbol{p}-\boldsymbol{p}_{j}\right)}
$$

with the kernel function for path loss measurements defined as

$$
\mathrm{K}_{p}(x)=\frac{1}{\left(2 \pi h_{p}^{2} \sigma_{p}^{2}\right)^{\frac{m}{2}}} \exp \left(-\frac{x^{T} x}{2\left(h_{p} \sigma_{p}\right)^{2}}\right),
$$

where $m$ is the number of base stations making measurements. For nonparametric TDoA location estimation, the calculation is

$$
\hat{\boldsymbol{\theta}}_{t}=\frac{\sum_{j=1}^{n} \boldsymbol{\theta}_{j} \mathrm{~K}_{t}\left(\boldsymbol{t}-\boldsymbol{t}_{j}\right)}{\sum_{j=1}^{n} \mathrm{~K}_{t}\left(\boldsymbol{t}-\boldsymbol{t}_{j}\right)}
$$

with the kernel function for TDoA measurements defined as

$$
\mathrm{K}_{t}(x)=\frac{1}{(2 \pi)^{\frac{m-1}{2}}\left|\boldsymbol{C}_{t}\right|^{\frac{1}{2}}} \exp \left(-\frac{\boldsymbol{x}^{T} \boldsymbol{C}_{t}^{-1} \boldsymbol{x}}{2}\right)
$$

with $C_{t}=h_{t}^{2} \sigma_{\tau}^{2} G G^{T}$. The constants $h_{p}$ and $h_{t}$ are defined as the kernel width constants. The effects of varying values of the constants is examined in Section 4 where it is shown that the estimators are robust to variations of the constants' values. It has been shown in the author's previous work that the nonparametric technique is also robust to the number of survey points with a graceful degradation of performance as the number of survey points is reduced [12], [13].

Using the kernel functions above and the survey data set, it is possible to calculate estimates of the covariances for the estimates using (22) from Appendix A. The covariance estimate for path loss based location estimates is denoted $\boldsymbol{R}_{p}$ and the covariance estimate for TDoA-based location estimates is denoted $R_{t}$. It is possible to calculate estimates for the mobile terminal locations using path loss and TDoA measurements in parallel obtaining $\hat{\boldsymbol{\theta}}_{p}, \boldsymbol{R}_{p}, \hat{\boldsymbol{\theta}}_{t}$, and $\boldsymbol{R}_{t}$. We now address the problem of fusing the estimates $\hat{\boldsymbol{\theta}}_{p}$ and $\hat{\boldsymbol{\theta}}_{t}$ to obtain a combined estimate $\theta$ with superior error covariance properties.

One standard approach in data fusion is to linearly combine the estimated values of the parameter of interest, in our case mobile terminal location, obtained from different measurements. We call this method estimate fusion. We define the dual estimate vector as $\hat{\boldsymbol{\theta}}_{k}=$ $\left[\hat{\boldsymbol{\theta}}_{p}^{T} \hat{\boldsymbol{\theta}}_{t}^{T}\right]^{T}$ and the dual location vector as $\boldsymbol{\theta}_{k}=\left[\boldsymbol{\theta}^{T} \boldsymbol{\theta}^{T}\right]^{T}$. The estimate fusion calculation is given by $\hat{\boldsymbol{\theta}}=\boldsymbol{W} \hat{\boldsymbol{\theta}}_{k}$, where $W$ is a weight matrix. For valid linear combinations, all the rows of $W$ sum to unity and $W$ is a full rank matrix. The standard data fusion approach is to calculate $W$ such that the trace, 
sum of the diagonal elements, for the covariance matrix of the fused estimate is minimized. The weight for each individual measurements estimate is based on its covariance. The estimate for a given measurement type will be given a higher weight if its covariance is low. The optimal $W$, which gives the lowest covariance is given by [33]:

$$
W=C_{k}^{-1} A^{T}\left(A C_{k}^{-1} A^{T}\right)^{-1}
$$

with

$$
\left.\boldsymbol{C}_{k}=\mathrm{E}\right]\left\{\left[\boldsymbol{\theta}_{k}-\hat{\boldsymbol{\theta}}_{k}\right]\left[\boldsymbol{\theta}_{k}-\hat{\boldsymbol{\theta}}_{k}\right]^{T} \mid \boldsymbol{p}, \boldsymbol{t}\right\}=\left[\begin{array}{cc}
\boldsymbol{R}_{p} & \boldsymbol{R}_{p t} \\
\boldsymbol{R}_{p t}^{T} & \boldsymbol{R}_{t}
\end{array}\right]
$$

and

$$
A=\left[\begin{array}{llll}
1 & 0 & 1 & 0 \\
0 & 1 & 0 & 1
\end{array}\right]
$$

$R_{p t}$ is the cross covariance of the path loss and TDoA measurement estimates,

$$
\boldsymbol{R}_{p t}=\mathrm{E}\left\{\left[\boldsymbol{\theta}_{p}-\hat{\boldsymbol{\theta}}_{p}\right]^{T}\left[\boldsymbol{\theta}_{t}-\hat{\boldsymbol{\theta}}_{t}\right] \mid \boldsymbol{p}, \boldsymbol{t}\right\} .
$$

his method assumes that covariance is the dominant factor in the error of the estimators compared to the bias and, thus, covariance reduction of the fused estimator is the most productive method of reducing error. The weight computation in (8) is only optimal if $\hat{\boldsymbol{\theta}}_{p}$ and $\hat{\boldsymbol{\theta}}_{t}$ are jointly Gaussian. These requirements make the optimality of the linear combination estimate fusion method difficult to prove for a given field application. The method is robust, however, in that linear combination using $W$ calculated using (8) with the true covariance matrices will always result in a fused estimator with lower covariance than the individual estimators. It must be noted that if the location estimates are non-Gaussian, their covariance and means are no longer sufficient statistics to describe their probability density functions, i.e., the mean and covariance of the location estimates no longer provide complete information about the distribution of the location estimates.

In practice, the covariances $R_{p}, R_{t}$, and $R_{p t}$ can only be estimated and the effectiveness of this approach in reducing the covariance of the final estimate is dependent on the accuracy of the covariance estimates. If the values in these matrices are highly inaccurate, it is possible for the fused estimate to have a covariance that is elevated over one of the individual estimators covariances. To calculate $R_{p t}$, the estimators for each measurement type cannot be run in parallel as they must exchange information to calculate the cross covariance matrix estimate. For the results on estimate fusion, we explore the possibilities for parallel estimation using each measurement type and, thus, assume that the location estimate error for each measurement type is independent in the data fusion calculation. To this end, the covariance matrix in the weight calculation (8) is set as

$$
\boldsymbol{C}_{k}=\left[\begin{array}{l|l}
\boldsymbol{R}_{p} & \\
\hline & \boldsymbol{R}_{t}
\end{array}\right]
$$

with $R_{p}$ and $R_{t}$ being the estimated covariance matrices from the location estimates for the path loss and TDoA measurements, respectively, and the off-diagonal blocks of $C_{k}$ contain all zeroes. The main advantage of parallel estimation for each measurement type is the independence of the data fusion method from the location estimate calculation for each measurement type. The nonparametric MMSE estimation calculations in (4) and (6) can be replaced with another location estimate calculation with an associated error covariance estimate and the data fusion technique can still be applied. For example, the nonparametric estimation using the path loss measurement vector could be replaced with the parametric estimator described in [31].

In the next part of this paper, we will present a location calculation that uses both measurement vectors simultaneously. This calculation does not have the optimality requirements for the calculations described in this section. By comparing the results of both methods in Section 4, we will see what the impact of the bias and independence assumptions is on the final location accuracy.

\subsection{Estimation of $\theta$ In a Single Calculation}

This section describes the calculation of $\hat{\boldsymbol{\theta}}$ using both path loss and TDoA measurement vectors in a single computation. To compute the location estimate using the combined measurement vector, $z=\left[\begin{array}{ll}p^{T} \boldsymbol{t}^{T}\end{array}\right]^{T}$, using (3) requires knowledge of the joint conditional pdf $\mathrm{f}(\boldsymbol{p}, \boldsymbol{t} \mid \boldsymbol{\theta})$. Parametric models for this conditional joint pdf are difficult to obtain, as was explained in the previous section.

The solution proposed in this paper is to use the survey data to calculate an approximation to the joint condition pdf. Using the calculated joint conditional pdf, a nonparametric MMSE estimation technique is used to calculate a location estimate using both the path loss measurement vector, $p$, and TDoA measurement vector, $t$, simultaneously.

The same survey set is used for the calculation described in this section as the calculation in Section 3.1. Following the derivation of the nonparametric MMSE estimator in Appendix $\mathrm{A}$, the joint pdf of $\theta, p$, and $t$ is approximated as [34]

$$
\mathrm{f}(\boldsymbol{\theta}, \boldsymbol{p}, \boldsymbol{t}) \approx \frac{1}{n} \sum_{j=1}^{n} \mathrm{~K}_{\theta, p, t}\left(\boldsymbol{\theta}-\boldsymbol{\theta}_{j}, \boldsymbol{p}-\boldsymbol{p}_{j}, \boldsymbol{t}-\boldsymbol{t}_{j}\right),
$$

where $n$ is the number survey points with $\boldsymbol{p}_{j}$ matching $\boldsymbol{p}$. The generalized joint kernel function is a general form multivariate pdf. The advantage of the use of the generalized joint kernel functions is that the joint pdf of the measurement and location vectors is well-approximated with a sum containing fewer terms. In a real-world application, this corresponds to the need for fewer survey points. The trivial ideal case being the approximation of the joint pdf with only one kernel function, the true joint pdf, and no survey data being needed. To use generalized kernel functions requires knowledge of the dependence between the $\theta, p$, and $t$ vectors prior to the application of the pdf approximation technique. Since we do not have this knowledge, we use the special kernel function, which is a pdf with $\theta, p$, and $t$ as independent, and the dependence in the true joint pdf is "learned" from the survey data. We decompose the joint kernel function into a product of kernel functions for each vector using a different kernel function and kernel width constant for each vector type. This method has been shown to be capable of approximating multidimensional pdfs with complex dependencies between the vectors being measured [35]. This makes the nonparametric approximate joint pdf: 


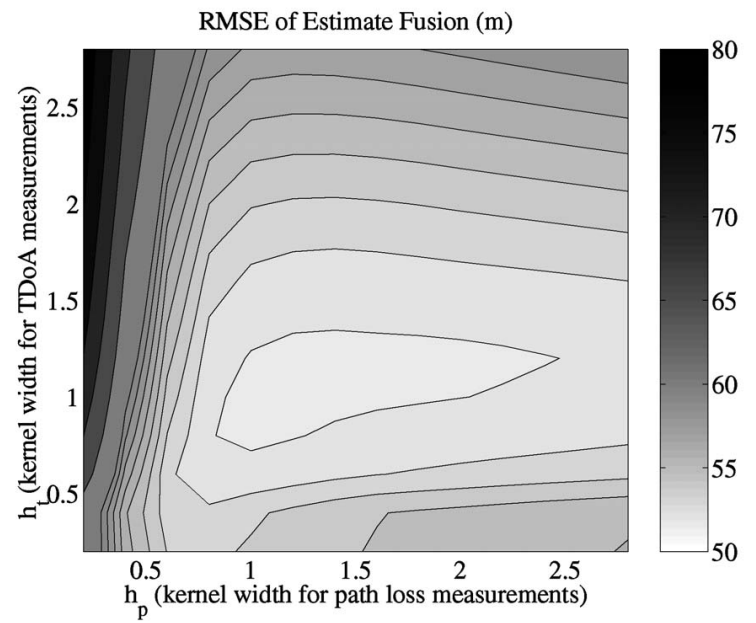

Fig. 3. Kernel widths for estimate fusion $\left(\sigma_{p}=6 \mathrm{~dB}, \sigma_{\tau}=15.0 \mathrm{~m}\right)$.

$$
\begin{aligned}
\mathrm{f}(\boldsymbol{\theta}, \boldsymbol{p}, \boldsymbol{t}) \approx & \frac{1}{n} \sum_{j=1}^{n}\left[\mathrm{~K}_{\theta}\left(\frac{\boldsymbol{\theta}-\boldsymbol{\theta}_{j}}{h_{\theta}}\right) \mathrm{K}_{p}\left(\frac{\boldsymbol{p}-\boldsymbol{p}_{j}}{h_{p}}\right)\right. \\
& \left.\times \mathrm{K}_{t}\left(\frac{\boldsymbol{t}-\boldsymbol{t}_{j}}{h_{t}}\right)\right] .
\end{aligned}
$$

$\mathrm{K}_{\theta}(\cdot), \mathrm{K}_{p}(\cdot)$, and $\mathrm{K}_{t}(\cdot)$ are the kernel functions. The constants $h_{\theta}, h_{p}$, and $h_{t}$ are the constants defining the kernel widths.

If we proceed using the approximate joint pdf defined in (14), the estimator for $\theta$ using both available measurement vectors is

$$
\hat{\boldsymbol{\theta}}=\sum_{j=1}^{n} \boldsymbol{\theta}_{j} \mathrm{w}_{j}(\boldsymbol{p}, \boldsymbol{t}),
$$

where

$$
\mathrm{w}_{j}(\boldsymbol{p}, \boldsymbol{t})=\frac{K_{p}\left(\frac{\boldsymbol{p}-\boldsymbol{p}_{j}}{h_{p}}\right) K_{t}\left(\frac{\boldsymbol{t}-\boldsymbol{t}_{j}}{h_{t}}\right)}{\sum_{i=1}^{n} K_{p}\left(\frac{\boldsymbol{p}-\boldsymbol{p}_{j}}{h_{p}}\right) K_{t}\left(\frac{\boldsymbol{t}-\boldsymbol{t}_{j}}{h_{t}}\right)} .
$$

The estimated covariance of a location estimate can be calculated using

$$
\boldsymbol{R}=\operatorname{Cov}(\hat{\boldsymbol{\theta}}) \approx\left[\sum_{j=1}^{n} \boldsymbol{\theta}_{j} \boldsymbol{\theta}_{j}^{T} w_{j}(\boldsymbol{z})\right]-\hat{\boldsymbol{\theta}} \hat{\boldsymbol{\theta}}^{T} .
$$

In the next section, it will be shown that this estimator is robust to variation of the kernel width constants, $h_{p}$ and $h_{t}$.

The single calculation estimator has the advantage that it does not require estimation of the error covariances. It also does not make any assumptions on the form of the pdf of the estimators. This estimator does not require more calculations than the estimator described in Section 3.1, with both estimators requiring the kernel functions to be evaluated the same number of times and adding up the same number of terms.

\section{Results}

The estimators are evaluated in a simulated urban environment with the propagation measurement model described in Section 2.1 using the propagation model parameter values provided in Table 3 . For each single set

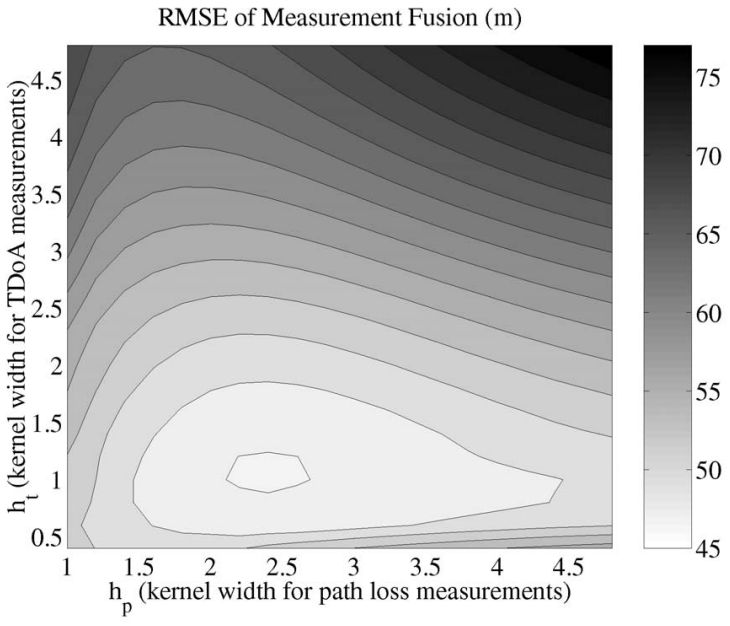

Fig. 4. Kernel widths for measurement fusion $\left(\sigma_{p}=6 \mathrm{~dB}, \sigma_{\tau}=15.0 \mathrm{~m}\right)$.

of parameter values, we simulate 10,000 Monte Carlo trials. In each trial, the true mobile terminal position is sampled from a uniform distribution over a single cell, simulated measurement vectors generated, and location estimates calculated from the simulated measurement vectors. The figure of merit used to evaluate the location estimators is the Root Mean Square Error, which is the square root of the sample mean of the square error of the location error, calculated using:

$$
R M S E=\sqrt{\sum_{k=1}^{N} \frac{1}{N}\left[\left(\hat{x}_{k}-x_{k}\right)^{2}+\left(\hat{y}_{k}-y_{k}\right)^{2}\right]}
$$

where $N$ is the total number of trials, $\left(x_{k}, y_{k}\right)$ is the mobile terminal location in the $k$ th simulated trial and $\left(\hat{x}_{k}, \hat{y}_{k}\right)$ is the estimated mobile terminal location for the $k$ th trial. The simulation software can be obtained from the authors upon request.

The first set of simulation were performed to calculate the optimal values of the kernel width constants $h_{t}$ and $h_{p}$ and to test the robustness of the estimators if suboptimal values are used for these constants. The results are shown as contour plots of the RMSE of the fused estimators as the kernel width constants are varied. The plot for estimate fusion of separate estimator results using the method described in Section 3.1 is shown in Fig. 3. The plot for measurement fusion using the method from Section 3.2 is shown in Fig. 4. The measurement noise values for the simulations generating these plots are set to $\sigma_{p}=6.0 \mathrm{~dB}$ and $\sigma_{\tau}=15 \mathrm{~dB}$, which match midrange values for typical urban propagation conditions. These plots show that both types of data fusion methods are robust to variations of the kernel width parameters with large regions in both plots having RMSE values almost as low as the to the optimal value. The measurement fusion calculation is more robust with less increase in the RMSE as the kernel width values vary from the optimal values. The optimal kernel width values for estimate fusion are $h_{p}=1.2$ and $h_{t}=1.0$. The optimal kernel width values for the measurement fusion calculation are $h_{p}=2.3$ and $h_{t}=1.0$.

The next set of simulations were performed to find the performance of location estimators using only one 


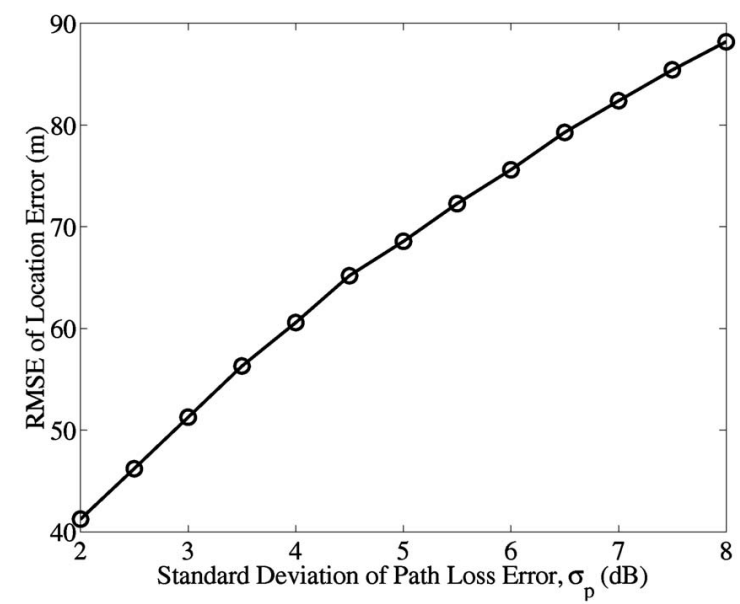

Fig. 5. RMSE of RSS estimator $\left(h_{p}=1.2\right)$.

measurement type. The optimal kernel width values for the estimate fusion method used. Fig. 5 shows the RMSE for the path loss-based estimation calculation described by (4) with $h_{p}=1.2$. Note that the estimator gives good performance even with large magnitude measurement noise and despite the $1 \mathrm{~dB}$ quantization. Fig. 6 shows the RMSE with the TDoA based estimation calculation described by (6) with $h_{t}=1.0$. The RMSE is a function of both path loss measurement noise and synchronization error since the path loss measurement noise influences the measuring base station selection process. Again, the estimator is robust to measurement noise variation and gives good results despite the $60 \mathrm{~m}$ quantization.

The improvement of the data fusion estimators over estimators using only one single measurement type are demonstrated in the next set of simulation results. For fixed values of $\sigma_{p}$ and $\sigma_{\tau}$, the percentage improvement in the RMSE from the data fusion method under consideration over the best RMSE obtained from the estimators using either path loss or TDoA measurements alone is calculated. Fig. 7 shows contour plots of the improvement from estimate fusion described in Section 3.1. Fig. 8 shows the improvement of the measurement fusion method. A comparison of

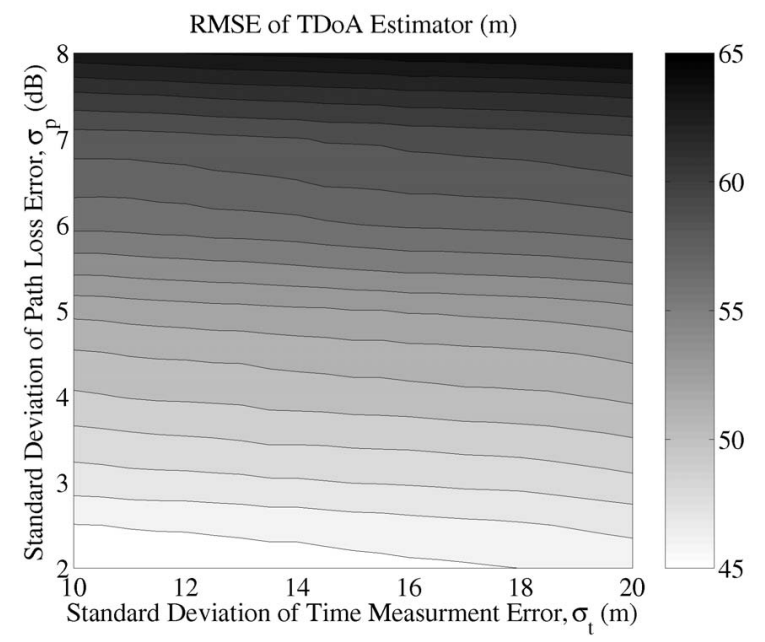

Fig. 6. RMSE of TDoA estimator $\left(h_{t}=1.0\right)$.

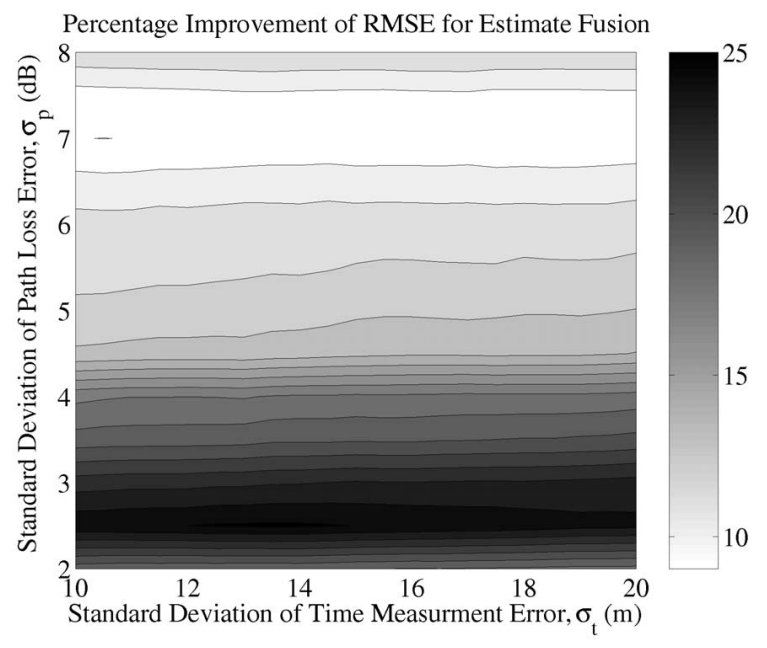

Fig. 7. RMSE improvement for estimate fusion $\left(h_{p}=1.2, h_{t}=1.0\right)$.

Fig. 7 and Fig. 8 shows that the measurement fusion method provides the greatest improvement in RMSE of the two data fusion methods. The minimum improvements from using data fusion is a reduction of RMSE by about 9 percent and a maximum improvement seen of about 26 percent for the range of measurement noise values tested.

The last set of simulations compares the cumulative distribution function (cdf) for the error distance for the location estimators. This function gives the probability that the error distance, defined as the distance from the estimated location to the true location, is less than a certain value. The error distance cdf for each of the estimators is calculated from 10,000 Monte Carlo trials for each of the estimators and plotted in Fig. 9. The environment parameters are set to midrange values for urban microcell propagation $\left(\sigma_{p}=6.0 \mathrm{~dB}\right.$ and $\left.\sigma_{\tau}=15.0 \mathrm{~m}\right)$. The values of the kernel width constants are set to the optimal values for each estimator described in the preceding paragraphs. The FCC requirements for location of mobile terminals for handset-based location technologies, the FCC's most stringent requirement, is also plotted [36]. If the cdf for a location estimator is above the FCC requirements line for all

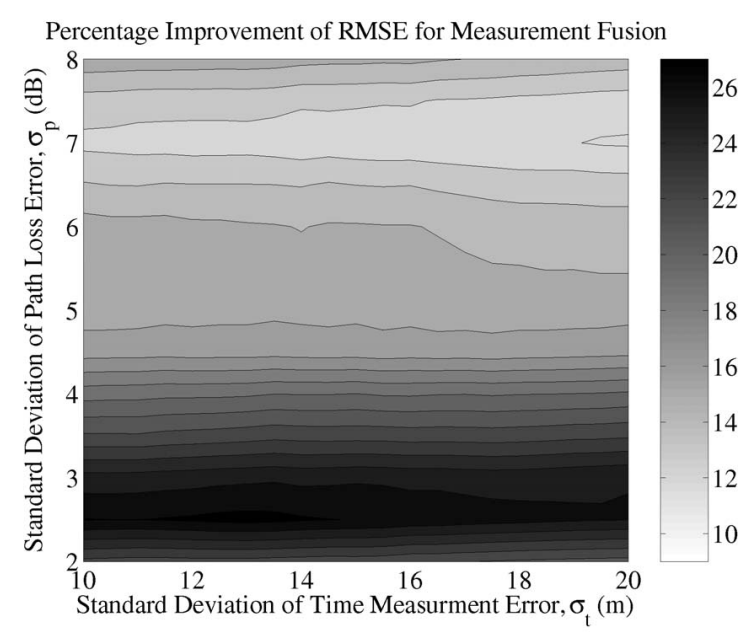

Fig. 8. RMSE improvement for measurement fusion $\left(h_{p}=2.3, h_{t}=1.0\right)$. 


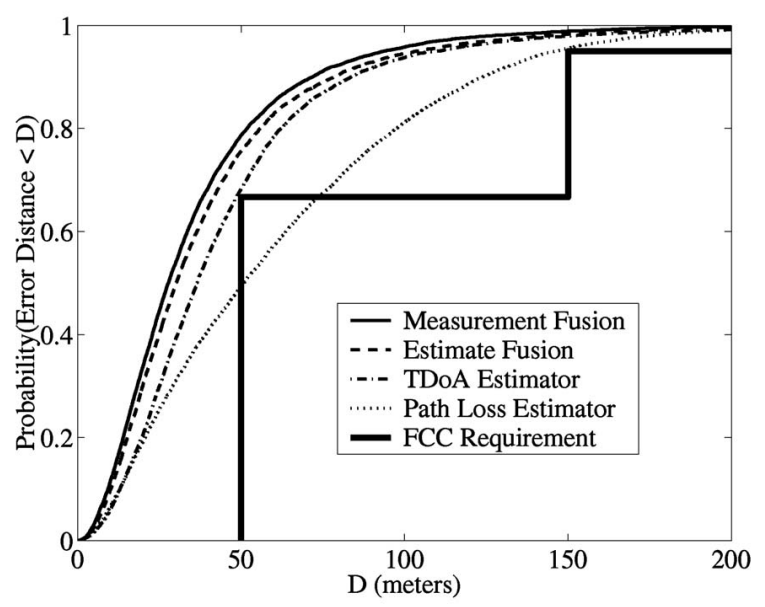

Fig. 9. Cumulative distribution function for error distances $\left(\sigma_{p}=6.0 \mathrm{~dB}\right.$, $\left.\sigma_{\tau}=15 \mathrm{~m}\right)$.

distances, the location estimator satisfies the requirement. These results show that the simulated measurement fusion location estimator provides the best performance of all estimators considered in this paper with a better cdf for all error distances.

\section{Conclusions}

These results support two sets of conclusions regarding choice of data fusion technique depending on the desire of the network operator and the feasibility of parallel computation of location estimates for path loss and TDoA measurements:

- The measurement fusion technique only gives a small improvement over the estimate fusion technique. This shows that the violation of the independence assumption of the location estimate's error for different measurement types for the estimate fusion technique causes only a small loss of performance. If the location estimates for different measurement types can be performed in parallel with the estimate fusion calculation performed afterword, this result shows that performing these calculation and assuming zero cross covariance of the location estimates will give good location accuracy. The parallel implementation results in a time savings over the measurement fusion calculation.

- If parallel implementation of location estimates for different measurement types is not possible and serial computation is performed, then using a measurement fusion technique will give superior accuracy and not require many more calculations.

There are several areas in which this work could be expanded in the future. The lower bound calculations from [12] can be expanded to bound location estimation using these data fusion methods. The use of the data fusion estimators with dynamic filters can also be explored with the possibility of different measurement types being available at different times.

It is our hope to be able to evaluate this method in field tests. This paper has shown that the nonparametric estimation technique can give good performance for location estimation in a simulated urban environment with complex propagation effects. The nonparametric technique described in this paper does not assume any propagation model and is robust to changes in the noise variances and selection of kernel parameters. This suggests the method will give good performance in other environments. The major factor which determines the accuracy of location estimates is the pdf of the measurement noise in that it determines how much information is available from the measurements about mobile terminal location. If the dependence of the measurement noise between base stations or between different measurement types is increased, the accuracy of the location estimates will be reduced as less information about location is present in the measurements. In this paper, we used measurement noise pdfs that are as close as possible to the true pdfs so accuracy measures close to field implementations' accuracy measures are obtained.

\section{APPEndiX A}

\section{NONPARAMETRIC MMSE Estimation}

It has been shown elsewhere that the estimator of a parameter $\theta$ based on measurements $z$ with the lowest Mean Square Error (MSE) is given by $\hat{\boldsymbol{\theta}}_{z}=\mathrm{E}[\boldsymbol{\theta} \mid z]$, where $\mathrm{E}[A \mid B]$ specifies the expected value of $A$ given $B$ [30]. This estimator is called the Minimum Mean Square Error (MMSE) estimator. Expansion of the expectation operator of the MMSE estimator results in

$$
\hat{\boldsymbol{\theta}}_{z}=\int \boldsymbol{\theta} \mathrm{f}(\boldsymbol{\theta} \mid z) d \boldsymbol{\theta},
$$

where $\mathrm{f}(\boldsymbol{\theta} \mid z)$ is the conditional pdf of the parameter given the measurements and the integration is performed over the domain of $\boldsymbol{\theta}$. A difficulty preventing real-world application of the MMSE estimator is that in many cases, the conditional pdf is not known. The solution often employed to avoid this difficulty is to use an approximate conditional pdf.

This appendix describes the implementation of the MMSE estimator using an approximate conditional pdf calculated based on survey data. This survey data set consists of a set of parameter values and measurement values $\left(\boldsymbol{\theta}_{j}, z_{j}\right)$ for $j=1,2, \ldots, n$, where $n$ is the number of survey points. $z_{j}$ is a measurement vector taken when the parameter vector was set to $\boldsymbol{\theta}_{j}$. It has been shown that an approximation to the joint pdf of $\theta$ and $z, \mathrm{f}(\theta, z)$, is

$$
\mathrm{f}(\boldsymbol{\theta}, z) \approx \frac{1}{n} \sum_{j=1}^{n} \mathrm{~K}_{\theta}\left(\boldsymbol{\theta}-\boldsymbol{\theta}_{j}\right) \mathrm{K}_{z}\left(z-z_{j}\right),
$$

where $\mathrm{K}_{\theta}(\cdot)$ and $\mathrm{K}_{z}(\cdot)$ are suitably selected kernel functions [37]. A convenient choice for the kernel functions are the pdfs of zero mean vector functions[34].

If we insert the approximate pdf from (20) into (19), the following approximation to the MMSE is obtained:

$$
\begin{aligned}
\hat{\boldsymbol{\theta}}_{z} & \approx \frac{\int \boldsymbol{\theta} \sum_{j=1}^{n} \mathrm{~K}_{\theta}\left(\boldsymbol{\theta}-\boldsymbol{\theta}_{j}\right) \mathrm{K}_{z}\left(z-z_{j}\right) d \boldsymbol{\theta}}{\int \sum_{j=1}^{n} \mathrm{~K}_{\theta}\left(\boldsymbol{\theta}-\boldsymbol{\theta}_{j}\right) \mathrm{K}_{z}\left(z-z_{j}\right) d \boldsymbol{\theta}} \\
& \approx \frac{\sum_{j=1}^{n} \boldsymbol{\theta}_{j} \mathrm{~K}_{z}\left(z-z_{j}\right)}{\sum_{j=1}^{n} \mathrm{~K}_{z}\left(z-z_{j}\right)} .
\end{aligned}
$$

The integrals of $\theta$ being solved using the fact that $\mathrm{K}_{\theta}(\cdot)$ is a zero mean pdf. This is referred to as a nonparametric 
technique since it does not rely on knowledge of any of the parameters of the joint pdf of $\theta$ and $z$ to calculate the estimate.

An advantage of this nonparametric estimator is that it is possible to also calculate an estimate of the covariance of the estimated parameter:

$$
\begin{aligned}
\operatorname{Cov}\left(\hat{\boldsymbol{\theta}}_{z}\right) & \approx \mathrm{E}\left[\boldsymbol{\theta} \boldsymbol{\theta}^{T} \mid z\right]-\hat{\boldsymbol{\theta}}_{z} \hat{\boldsymbol{\theta}}_{z}^{T} \\
& \approx\left[\frac{\sum_{j=1}^{n} \boldsymbol{\theta}_{j} \theta_{j}^{T} \mathrm{~K}_{z}\left(z-z_{j}\right)}{\sum_{j=1}^{n} \mathrm{~K}_{z}\left(z-z_{j}\right)}\right]-\hat{\boldsymbol{\theta}}_{z} \hat{\boldsymbol{\theta}}_{z}^{T} .
\end{aligned}
$$

\section{APPENDIX B}

\section{Transforming Measurement Vectors}

This appendix gives the method of converting $\boldsymbol{p}_{j}$ and $\boldsymbol{t}_{j}$ when $\boldsymbol{b}_{j}$ matches $\boldsymbol{b}$, in that all the entries of $\boldsymbol{b}$ are found in $\boldsymbol{b}_{j}$, but $b_{j} \neq \boldsymbol{b}$. To properly compare $\boldsymbol{t}$ with $\boldsymbol{t}_{j},[\boldsymbol{t}]_{k}$, and $\left[\boldsymbol{t}_{j}\right]_{k}$ must be derived from measurements from measurements from the same base stations for all $k \in\{1,2, \ldots, m-1\}$, where $m$ is the number of base stations making measurements. We denote the transformed measurement vectors as $\boldsymbol{b}_{j}^{\prime}, \boldsymbol{p}_{j}^{\prime}$, and $t_{j}^{\prime}$, respectively. The reorder matrix $T$ is defined from $b_{j}^{\prime}=T b_{j}=b$, where each row of $T$ contains a single one with all other entries being zero, i.e., $T$ is obtained by swapping rows of an appropriately sized identity matrix. Using $T, p_{j}^{\prime}=T p_{j}$.

Unfortunately, the transformation of $t_{j}$ is not so simple. Ignoring quantization, we will denote as $\tau_{j}$ the ToA pseudomeasurement vector that is the source of $t_{j}=G \tau_{j}$. $\left[\tau_{j}\right]_{k}$ is the raw ToA propagation distance measurement for the base station indexed by the $k$ th entry of $\boldsymbol{b}_{j}$. If $\tau_{j}$ were available, then the transformed TDoA vector could be obtained using $t_{j}^{\prime}=G T \tau_{j}$. We define $F$ such that $t_{j}^{\prime}=F t_{j}$. This gives us:

$$
\begin{aligned}
& F t_{j}=t_{j}^{\prime} \\
& F G \tau_{j}=G T \tau_{j} \\
& F G=G T \\
& F G G^{+}=G T G^{+} \\
& F=G T G^{+},
\end{aligned}
$$

where $G^{+}$is the pseudoinverse of $G[38]$. Thus, $t_{j}^{\prime}=G T G^{+} t_{j}$.

\section{ACKNOWLEDGMENTS}

This work was supported by the Nortel Institute for Telecommunications. Some of this work was presented at the 2003 PACRIM conference.

\section{REFERENCES}

[1] L. Jorguseski, E. Fledderus, J. Farserotu, and R. Prasad, "Radio Resource Allocation in Third-Generation Mobile Communication Systems," IEEE Comm. Magazine, vol. 39, no. 2, pp. 117-123, Feb. 2001.

[2] T. Lewis, "Tracking the 'Anywhere Anytime' Inflection Point," Computer, vol. 32, no. 2, pp. 134-136, Feb. 2000.

[3] J. Caffery Jr. and G. Stuber, "Subscriber Location in CDMA Cellular Networks," IEEE Trans. Vehicular Technology, vol. 47, no. 2, pp. 406-416, May 1998.
[4] N. Bulusu, J. Hedemann, and D. Estrin, "GPS-Less Low-Cost Outdoor Localization for Very Small Devices," IEEE Personal Comm., vol. 7, no. 5, pp. 28-34, Oct. 2000.

[5] Y. Chan and K. Ho, "A Simple and Efficient Estimator for Hyperbolic Location," IEEE Trans. Signal Processing, vol. 42, no. 8, pp. 1905-1915, Aug. 1994.

[6] J. Reed, K. Krizman, B. Woerner, and T. Rappaport, "An Overview of the Challenges and Progress in Meeting the E-911 Requirement for Location Service," IEEE Comm. Magazine, vol. 36, no. 4, pp. 3037, Apr. 1998.

[7] S. Soliman and C. Wheatley, "Geolocation Technologies and Applications for Third Generation Wireless," Wireless Comm. and Mobile Computing, vol. 2, no. 3, pp. 229-251, May 2002.

[8] T. Kerr, "A Critical Perspective on Some Aspects of GPS Development and Use," Proc. AIAA/IEEE Digital Avionics Systems Conf., pp. 9.4-9-9.4-20, Oct. 1997.

[9] P.-C. Chen, "A Cellular Based Mobile Location Tracking System," Proc. IEEE Vehicular Technology Conf., pp. 1979-1983, May 1999.

[10] M. Spirito and M. Wylie-Green, "Mobile Station Location in Future TDMA Mobile Communication Systems," Proc. IEEE Fall Vehicular Technology Conf., pp. 790-794, Sept. 1999.

[11] M. Wylie-Green and S. Wang, "Observed Time Difference (OTD) Estimation for Mobile Positioning in IS-136 in the Presence of BTS Clock Drift," Proc. Fall Vehicular Technology Conf., pp. 2677-2681, 2001.

[12] M. McGuire, K. Plataniotis, and A. Venetsanopoulos, "Location of Mobile Terminals Using Time Measurements and Survey Points," IEEE Trans. Vehicular Technology, vol. 52, no. 4, pp. 1012-1031, July 2003.

[13] M. McGuire and K. Plataniotis, "Estimating Position of Mobile Terminal from Path Loss Measurements with Survey Data," Wireless Comm. and Mobile Computing, vol. 3, no. 1, pp. 51-62, Feb. 2003.

[14] T. Roos, P. Myllymaki, H. Tirri, P. Misikangas, and J. Sievanen, "A Probabilistic Approach to WLAN User Location Estimation," Int'l J. Wireless Information Networks, vol. 9, no. 3, pp. 155-164, July 2002.

[15] D. Fox, J. Hightower, L. Liao, D. Schulz, and G. Borriello, "Bayesian Filtering for Location Estimation," IEEE Pervasive Computing, vol. 2, no. 3, pp. 24-33, July-Sept. 2003.

[16] S. Ahonen and P. Eskelinen, "Mobile Terminal Location for UMTS," IEEE Aerospace and Electronic Systems Magazine, vol. 18, no. 2, pp. 23-27, Feb. 2003.

[17] D. Hall and J. Llinas, "An Introduction to Multisensor Data Fusion," Proc. IEEE, vol. 85, no. 1, pp. 6-25, 1997.

[18] L. Cong and Z. Weihua, "Hybrid TDOA/AOA Mobile User Location for Wideband CDMA Cellular Systems," IEEE Trans. Wireless Comm., vol. 1, no. 5, pp. 439-447, 2002.

[19] Z. Gu and E. Gunawan, "Radiolocation in CDMA Cellular System Based on Joint Angle and Delay Estimation," Wireless Personal Comm., vol. 23, no. 3, pp. 297-309, Dec. 2002.

[20] T. Kleine-Ostmann and A. Bell, "A Data Fusion Architecture for Enhanced Position Estimation in Wireless Networks," IEEE Comm. Letters, vol. 5, no. 8, pp. 343-345, Aug. 2001.

[21] S. Merigeault, M. Batariere, and J. Patillon, "Data Fusion Based on Neural Network for the Mobile Subscriber Location," Proc. IEEE Fall Vehicular Technology Conf., pp. 536-541, Sept. 2000.

[22] N. Thomas, D. Cruickshank, and D. Laurenson, "Performance of a TDOA-AOA Hybrid Mobile Location System," Proc. Int'l Conf. $3 G$ Mobile Comm. Technologies, pp. 216-220, 2001.

[23] D. Torrieri, "Statistical Theory of Passive Location Systems," IEEE Trans. Aerospace and Electronic Systems, vol. 20, no. 2, pp. 183-198, Mar. 1984.

[24] P. Lundqvist, H. Asplund, and S. Fischer, "Evaluation of Positioning Systems," Technical Subcommtittee T1P1, Technical Report T1P1.5/97-110, Dec. 1997.

[25] L. Greenstein, V. Erceg, Y. Yeh, and M. Clark, "A New Path-Gain/ Delay-Spread Propagation Model for Digital Cellular Channels," IEEE Trans. Vehicular Technology, vol. 46, no. 2, pp. 477-485, May 1997.

[26] J.-E. Berg, "Building Penetration Loss along Urban Street Microcells," Proc. Personal, Indoor and Mobile Radio Communication Conf. (PIMRC), pp. 795-797, Oct. 1996.

[27] A. Goldsmith, L. Greenstein, and G. Foschini, "Error Statistics of Real Time Power Measurements in Cellular Channels with Multipath and Shadowing," IEEE Trans. Vehicular Technology, vol. 43, no. 3, pp. 439-446, Aug. 1994. 
[28] J. Parsons, The Mobile Radio Propagation Channel, 2nd ed. Toronto: John Wiley \& Sons, Inc., 2000.

[29] H. Gombachika and O. Tonguz, "Influence of Multipath Fading and Mobile Unit Velocity on the Performance of PN Tracking in CDMA Systems," Proc. IEEE Vehicular Technology Conf., pp. 22062209, May 1997.

[30] J. Mendel, Lessons in Estimation Theory for Signal Processing, Communications, and Control. New Jersey: Prentice Hall, 1995.

[31] T. Roos, P. Myllymaki, and H. Tirri, "A Statistical Modeling Approach to Location Estimation," IEEE Trans. Mobile Computing, vol. 1, no. 1, pp. 59-69, Jan.-Mar. 2002.

[32] P. Bernadin, M. Yee, and T. Ellis, "Cell Radius Inaccuracy: A New Measure of Coverage Reliability," IEEE Trans. Vehicular Technology, vol. 47, no. 4, pp. 1215-1226, Nov. 1998.

[33] Y. Zhu, Multisensor Decision and Estimation Fusion. Boston: Kluwer Academic Publishers, 2003.

[34] D. Scott, Multivariate Density Estimation: Theory, Practice, and Visualization. Toronto: John Wiley \& Sons, 1992.

[35] B. Silverman, Density Estimation for Statistics and Data Analysis. London: Chapman \& Hall, 1986.

[36] FCC, "OET Bulletin no. 71, Guidelines for Testing and Verifying the Accuracy of Wireless E911 Location Systems," Apr. 2000.

[37] R. Duda, P. Hart, and D. Stork, Pattern Classification, 2nd ed. Toronto: John Wiley \& Sons, Inc., 2001.

[38] S. Haykin, Adaptive Filter Theory. Upper Saddle River, New Jersey: Prentice Hall, 2002.

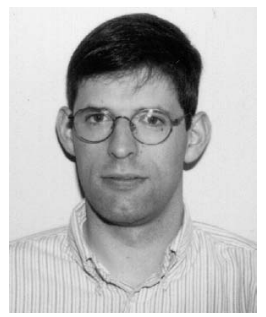

Michael McGuire received the BEng degree in computer engineering and the MASc degree from the University of Victoria in 1995 and 1997, respectively. He received the $\mathrm{PhD}$ degree from the Department of Electrical and Computer Engineering at the University of Toronto in 2003. He is presently an assistant professor in the Department of Electrical and Computer Engineering at the University of Victoria. His research interests are estimation and control algorithms for wireless cellular networks. He is a member of the IEEE and the IEEE Computer Society.

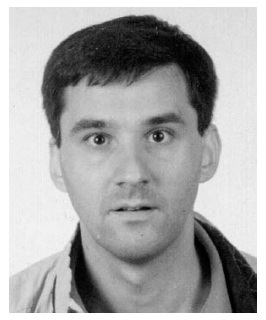

Konstantinos N. Plataniotis received the BEng degree in computer engineering from the Department of Computer Engineering and Informatics, University of Patras, Patras, Greece, in 1988 and the MS and PhD degrees in electrical engineering from the Florida Institute of Technology (Florida Tech), Melbourne, Florida, in 1992 and 1994, respectively. He was a research associate with the Computer Technology Institute (C.T.I), Patras, Greece, from 1989 to 1991 and a postdoctoral fellow at the Digital Signal \& Image Processing Laboratory, Department of Electrical and Computer Engineering, University of Toronto, from 1995 to 1997. From August 1997 to June 1999, he was an assistant professor with the School of Computer Science at Ryerson Polytechnic University. While at Ryerson, Professor Plataniotis served as a lecturer in 12 courses to industry and Continuing Education programs. Since 1999, he has been with the University of Toronto as an assistant professor at the Department of Electrical \& Computer Engineering where he researches and teaches adaptive systems and multimedia signal processing. He coauthored, with A.N. Venetsanopoulos, a book on Color Image Processing \& Applications, (Springer Verlag, May 2000, ISBN 3-540-66953-1), he is a contributor to three books, and he has published more than 100 papers in refereed journals and conference proceedings on the areas of adaptive systems, signal and image processing, and communication systems and stochastic estimation. He is a member of the IEEE Technical Committee on Neural Networks for Signal processing and the technical cochair of the Canadian Conference on Electrical and Computer Engineering, CCECE 2001, May 13-16, 2001, Toronto, Ontario. His current research interests include: adaptive systems statistical pattern recognition, multimedia data processing, statistical communication systems, and stochastic estimation and control. $\mathrm{He}$ is a senior member of the IEEE and the IEEE Computer Society.

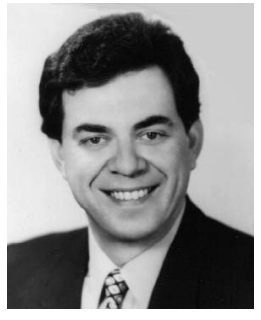

Anastasios N. Venetsanopoulos received the diploma in engineering degree from the National Technical University of Athens (NTU), Greece, in 1965, and the MS, MPhil, and PhD degrees in electrical engineering from Yale University in 1966, 1968, and 1969, respectively. He joined the Department of Electrical and Computer Engineering at the University of Toronto in September 1968 as a lecturer and he was promoted to assistant professor in 1970 , associate professor in 1973, and professor in 1981. Since July 1997, he has been associate chair: Graduate Studies of the Department of Electrical and Computer Engineering and was acting chair during the spring term of 1998-1999. In 1999, a chair in Multimedia was established in the ECE Department, made possible by a donation of $\$ 1.25 \mathrm{M}$ from Bell Canada, matched by $\$ 1.0 \mathrm{M}$ of university funds. Professor Venetsanopoulos assumed the position as Inaugural Chairholder in July 1999 and two additional assistant professor positions became available in the same area. He has served as chair of the Communications Group and associate chair of the Department of Electrical Engineering and associate chair: Graduate Studies for the Department of Electrical and Computer Engineering. He was on research leave at Imperial College of Science and Technology, the National Technical University of Athens, the Swiss Federal Institute of Technology, the University of Florence, and the Federal University of Rio de Janeiro, and has also served as adjunct professor at Concordia University. He has served as lecturer in 138 short courses to industry and continuing education programs and as consultant to numerous organizations; he is a contributor to 29 books, a coauthor of Nonlinear Filters in Image Processing: Principles Applications (ISBN-0-7923-9049-0), and Artificial Neural Networks: Learning Algorithms, Performance Evaluation and Applications (ISBN-0-79239297-3), Fuzzy Reasoning in Information Decision and Control Systems (ISBN-0-7293-2643-1), and Color Image Processing and Applications (ISBN-3-540-66953-1), and has published more than 680 papers in refereed journals and conference proceedings on digital signal and image processing and digital communications. Prof. Venetsanopoulos has served as chair on numerous boards, councils, and technical conference committees of the Institute of Electrical and Electronic Engineers (IEEE), such as the Toronto Section (1977-1979) and the IEEE Central Canada Council (1980-1982); he was president of the Canadian Society for Electrical Engineering and vice president of the Engineering Institute of Canada (EIC) (1983-1986). He was a guest editor or associate editor for several IEEE journals and the editor of the Canadian Electrical Engineering Journal (1981-1983). He is a member of the IEEE Communications, Circuits, and Systems, Computer, and Signal Processing Societies of IEEE, as well as a member of Sigma Xi, the Technical Chamber of Greece, the European Association of Signal Processing, the Association of Professional Engineers of Ontario (APEO) and Greece. He was elected as a fellow of the IEEE "for contributions to digital signal and image processing," he is also a fellow of the EIC, and was awarded an Honorary Doctorate from the National Technical University of Athens, in October 1994. In October 1996, he was awarded the "Excellence in Innovation Award" of the Information Technology Research Centre of Ontario and Royal Bank of Canada, "for innovative work in color image processing and its industrial applications." In November 2000, he became a recipient of the "Millennium Medal of IEEE." In April 2001, he became a fellow of the Canadian Academy of Engineering. Between July 2001 and June 2006, he will be the Dean of the Faculty of Applied Science and Engineering of the University of Toronto. He is a fellow of the IEEE and the IEEE Computer Society.

$\triangleright$ For more information on this or any other computing topic, please visit our Digital Library at www.computer.org/publications/dlib. 